\title{
Lipid Lateral Mobility in Cochlear Outer Hair Cells: Regional Differences and Regulation by Cholesterol
}

\author{
Louise E. Organ ${ }^{1}$ and Robert M. RaphaEl ${ }^{1}$ \\ ${ }^{1}$ Department of Bioengineering, Rice University, MS-142, PO Box 1892, Houston, TX 77251-1892, USA
}

Received: 30 May 2008; Accepted: 26 April 2009; Online publication: 11 June 2009

\begin{abstract}
The outer hair cell (OHC) lateral plasma membrane houses the transmembrane protein prestin, a necessary component of the yet unknown molecular mechanism (s) underlying electromotility and the exquisite sensitivity and frequency selectivity of mammalian hearing. The importance of the plasma membrane environment in modulating $\mathrm{OHC}$ electromotility has been substantiated by recent studies demonstrating that membrane cholesterol alters prestin activity in a manner consistent with cholesterol-induced changes in auditory function. Cholesterol is known to affect membrane material properties, and measurements of lipid lateral mobility provide a method to asses these changes in living OHCs. Using fluorescence recovery after photobleaching (FRAP), we characterized regional differences in the lateral diffusion of the lipid analog di-8-ANEPPS in OHCs and investigated whether lipid mobility, which reflects membrane fluidity, is sensitive to membrane cholesterol. FRAP experiments revealed quantitative differences in lipid lateral mobility among the apical, lateral, and basal regions of the $\mathrm{OHC}$ and demonstrated that diffusion in individual regions is uniquely sensitive to cholesterol manipulations. Interestingly, in the lateral region, both cholesterol depletion and loading significantly reduced the effective diffusion coefficient from control values. Thus, the fluidity of the $\mathrm{OHC}$ lateral plasma membrane is regulated by cholesterol levels in a non-monotonic manner, suggesting that the overall material properties of the lateral plasma membrane
\end{abstract}

Correspondence to: Robert M. Raphael - Department of Bioengineering - Rice University - MS-142, PO Box 1892, Houston, TX 772511892, USA. Telephone: +1-713-3483494; fax: +1-713-3485877; email: rraphael@rice.edu are optimally tuned for OHC function in the native state. These results support the idea that the cholesteroldependent regulation of prestin function and electromotility correlates with changes in the properties of the lipid environment that surrounds and supports prestin.

Keywords: fluorescence recovery after photobleaching (FRAP), di-8-ANEPPS, prestin, diffusion

\section{INTRODUCTION}

Since the discovery of the outer hair cell (OHC) transmembrane protein prestin, much work has focused on understanding its structure and function (Zheng et al. 2000; He et al. 2006; Ashmore 2008). Equally important, but less examined, is the role the plasma membrane environment plays in prestin activity and $\mathrm{OHC}$ electromotility. The relationship between the material (i.e., chemical, physical, and mechanical) properties of biological membranes and their physiologic function is an area of growing interest, and many membrane properties affect the function of diverse membrane proteins (Yeagle 1991; Cullis et al. 1996; Bastiaanse et al. 1997; Lundbaek et al. 2004; Janmey and Kinnunen 2006; Andersen and Koeppe 2007). As a notable recent example, changes in lipid composition and membrane tension were shown to alter the channel gating characteristics of voltage-dependent potassium channels (Schmidt and Mackinnon 2008). Thus, understanding the extent to which membrane-protein interactions regulate prestin function and electromotility is an important area of auditory research that requires characterizing the biophysical properties of the OHC plasma mem- 
brane. Ideally, this would include measurements of both molecular (e.g., lipid packing density, electrostatics, hydrophobic thickness, etc.) and bulk (e.g., fluidity, permeability, stiffness, etc.) properties of the membrane. Unfortunately, the few techniques available to directly measure these parameters are often limited, particularly in their application to the plasma membrane of living cells (Edidin 2003). However, quantifying the translational diffusion or lateral mobility of molecules in the plane of the bilayer is a useful method to directly measure membrane fluidity and to indirectly assess changes in other interconnected membrane material properties in live, native specimens (Axelrod et al. 1976; Edidin 1987, 1994; Cullis et al. 1996). Lateral mobility measurements are readily made using fluorescence recovery after photobleaching (FRAP) techniques. A distinct advantage of applying FRAP to measure lateral diffusion is the ability to provide a dynamic view of the membrane environment, as the fluidity is altered by membrane perturbations.

Previous work has measured lateral diffusion in OHCs using FRAP and demonstrated that the fluidity of the OHC plasma membrane is similar to other eukaryotic cells (Oghalai et al. 1999, 2000; de Monvel et al. 2006). Interestingly, lipid lateral mobility in OHCs has been reported to be both voltage- and tension-dependent (Oghalai et al. 2000). Additionally, lipid diffusion in the OHC has been reported to be anisotropic, faster in the axial than circumferential direction (de Monvel et al. 2006). This observation is attributed to the structure and orientation of the cortical actin-spectrin cytoskeleton (de Monvel et al. 2006) and/or the small, structural domains observed in the OHC plasma membrane (Zhang and Kalinec 2002). Recent work has also shown that intense noise exposure decreases lateral diffusion in the $\mathrm{OHC}$ plasma membrane, suggesting that alterations in membrane material properties are involved in the mechanism of noise damage (Chen and Zhao 2007). Despite these studies, our knowledge about the composition, organization, dynamics, and functional importance of lipid components in the OHC plasma membrane remains limited.

The characteristic material properties of biological membranes can vary in different regions of a cell or even between small domains within the membrane (Hilgemann 2003; Heidemann and Wirtz 2004). The OHC is a polarized cell and can be divided into apical, lateral, and basal regions, each specialized for different functions (Fig. 1A; Brownell et al. 2001; Santos-Sacchi 2003). While the apical region is separated from the basolateral region via a proteinaceous tight junction belt typical to epithelial cell types, there is not a similar physical separation between the lateral wall and basal pole (Zhang and Kalinec 2002). However, prestin
A

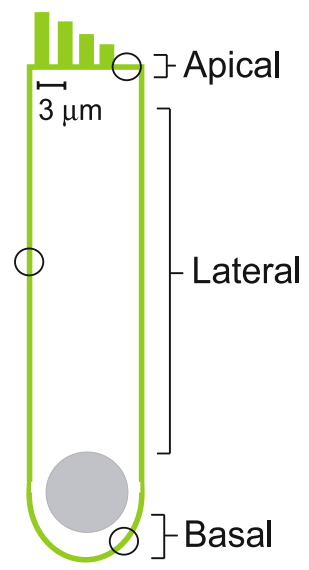

B
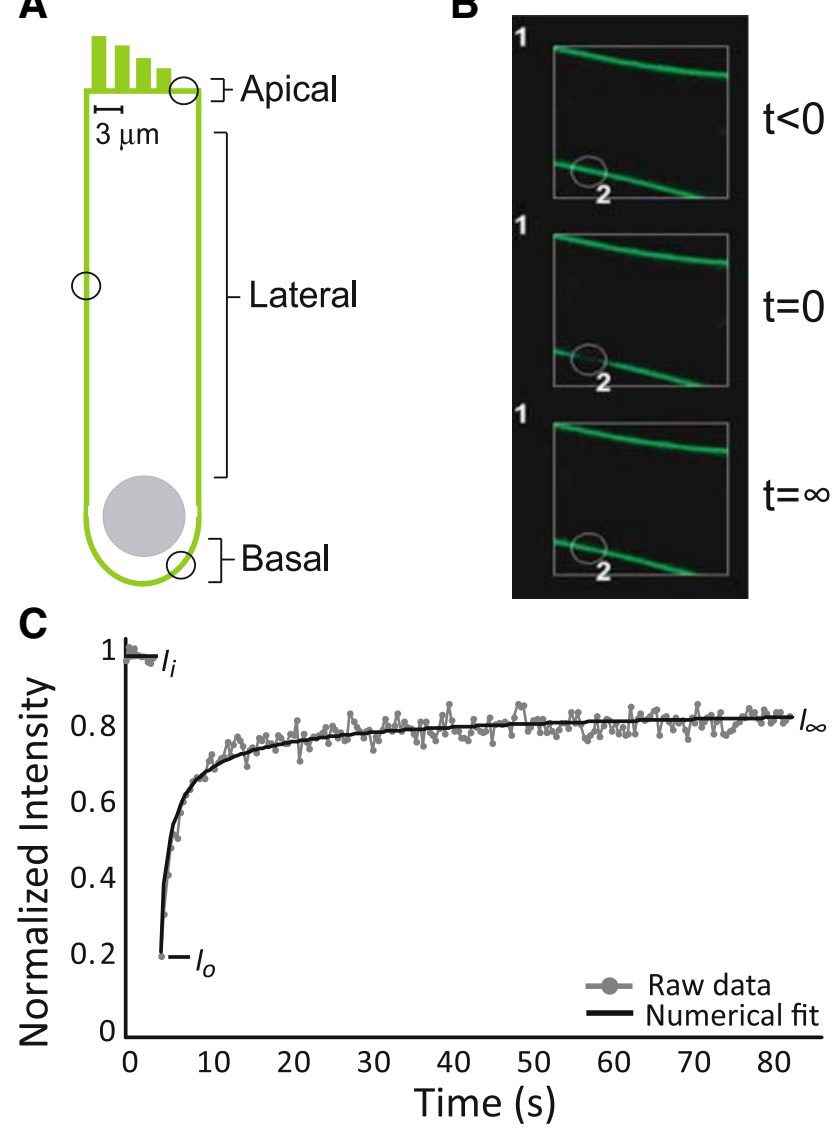

FIG. 1. A Depiction of an $\mathrm{OHC}$, with circles indicating possible areas for FRAP experimentation in the three regions: lateral, basal, and apical. B Representative FRAP experiment performed in an $\mathrm{OHC}$. The white square measures $14.3 \mu \mathrm{m}^{2}$, and the circle defining the bleach region (indicated by the number 2 ) is $\sim 3 \mu \mathrm{m}$ in diameter. C Sample FRAP recovery curve in an $\mathrm{OHC}$. From the raw data curve, a numerical fitting routine is used to determine the effective diffusion coefficient, $D$. Intensity values used to calculate the mobile fraction, $M_{\mathrm{f}}$, are noted. $I_{\mathrm{i}}$ is the initial, pre-bleach intensity calculated as the average of the first ten scans. $I_{0}$ is the intensity immediately postbleaching, and $I_{\infty}$ is the final, recovery intensity, equal to the average of the last ten scans.

appears to be unable to diffuse from the lateral wall to the basal pole, even upon degradation of the cytoskeleton (Takahashi and Santos-Sacchi 2001). Likewise, receptors and ion channels in the basal pole do not migrate to the lateral wall (Kalinec et al. 1992; Zhang and Kalinec 2002). Therefore, some other mechanism organizes and preserves the molecular composition of these membrane areas, and it is conceivable that the OHC actively maintains a distinct lipid composition in each of the three regions. We hypothesize that as a result of differences in membrane composition, bilayer material properties vary between $\mathrm{OHC}$ regions, and these variations can be assessed by measuring lipid lateral mobility via FRAP experiments.

Cholesterol is a crucial molecular component of mammalian plasma membranes and is an important 
regulator of membrane material properties including, but not limited to, lateral mobility, permeability, stiffness, mechanical moduli, lateral membrane organization, hydrophobic thickness, and lipid packing density (Needham and Nunn 1990; Yeagle 1991; Cullis et al. 1996; Mouritsen and Zuckermann 2004; McIntosh and Simon 2006; Andersen and Koeppe 2007). Recently, changes in cochlear cholesterol levels were shown to modulate the amplitude of distortion product otoacoustic emissions (DPOAEs), consistent with changes in electromotility (Rajagopalan et al. 2007). Directly altering membrane cholesterol levels in OHCs also reversibly shifted the nonlinear capacitance (NLC), the electrical signature associated with prestin function and electromotility (Santos-Sacchi 2003), suggesting that cholesterol levels dynamically tune prestin to operate at maximal gain near the $\mathrm{OHC}$ resting potential (Rajagopalan et al. 2007). These results are important in the context of other reports that hypercholesterolemia may be linked to hearing loss in both animal models and humans (Morizono and Paparella 1978; Morizono et al. 1985; Saito et al. 1986; Sikora et al. 1986; Preyer et al. 2001; Marcucci et al. 2005). However, the biophysical mechanisms by which cholesterol affects prestin function and hearing are unknown. Potentially, cholesterol levels could alter prestin activity by modifying the material properties of the membrane environment. To investigate this idea, we performed FRAP experiments and quantified changes in membrane fluidity by measuring the lateral mobility of di-8-ANEPPS, a lipid analog, in OHCs and human embryonic kidney (HEK) cells following cholesterol manipulations. We demonstrated variations in cholesterol-dependent lipid mobility among the $\mathrm{OHC}$ regions and showed that the fluidity of the lateral plasma membrane is uniquely responsive to cholesterol levels. These results highlight the complexity and physiological relevance of membraneprotein interactions and the importance of their inclusion in models of $\mathrm{OHC}$ electromotility.

\section{METHODS}

\section{$\mathrm{OHC}$ isolation and fluorescent labeling}

OHCs were obtained from adult, albino guinea pigs (Charles River, Wilmington, MA, USA). Females ranging in weight from 100 to $240 \mathrm{~g}$ were anesthetized with halothane (Halocarbon, River Edge, NJ, USA), decapitated, and both temporal bones were excised. The bones were bathed in standard extracellular solution (ECS; $140 \mathrm{mM} \mathrm{NaCl}, 5 \mathrm{mM} \mathrm{KCl}, 10 \mathrm{mM}$ HEPES, $10 \mathrm{mM}$ glucose, $2 \mathrm{mM} \mathrm{CaCl}_{2}, 1 \mathrm{mM} \mathrm{MgCl}_{2}, \mathrm{pH} 7.3$, and 290 $305 \mathrm{mOsm}$ ), and the bullae were opened to reveal the otic capsule, which was delicately chiseled away to expose the top two cochlear turns. Then, the entire bulla was placed in $3 \mathrm{~mL}$ of ECS loaded with $150 \mu \mathrm{M}$
di-8-ANEPPS (Pyridinium, 4-[2-[6-(dioctylamino)-2naphthalenyl]ethenyl]-1-(3-sulfopropyl)-) (Invitrogen, Carlsbad, CA, USA, cat. No. D-3167) and incubated at room temperature for $20 \mathrm{~min}$. All animal experiments and procedures were approved by Rice University's Institutional Animal Care and Use Committee (protocols A04091302 and A08082001).

Di-8-ANEPPS stock solutions were made at $1.69 \mathrm{mM}$ in dimethyl sulfoxide. The structure of di-8-ANEPPS is analogous to lipids, with a polar head region and a nonpolar tail region. This suggests that di-8-ANEPPS lateral mobility is similar to that of native constituent lipids, and di-8-ANEPPS cleanly and homogeneously labels the plasma membranes of both OHCs and HEKs (Oghalai et al. 1998, 1999, 2000; de Monvel et al. 2006; Chen and Zhao 2007; Greeson and Raphael 2007). While the use of DMSO as a carrier for di-8-ANEPPS may have some effect on the material properties of the OHC plasma membrane, all cells were treated identically with di-8-ANEPPS, and we do not believe that this treatment in any way predisposed OHCs to be abnormally sensitive to cholesterol manipulations. In addition, although membrane material properties are temperature-dependent, previous work demonstrated only moderate changes in the lateral mobility of di-8ANEPPS in OHCs between $22^{\circ} \mathrm{C}$ and $37^{\circ} \mathrm{C}$ (Oghalai et al. 1999).

After dye incubation, cochleae were bathed in fresh ECS, and OHCs were removed from the top two cochlear turns through mechanical dissociation. Specimens were allowed to settle on poly-D-lysine-coated 35-mm MatTek dishes (MatTek Corporation, Ashland, MA, USA, no. P35G-1.5-10-C) for 5-10 min before viewing. OHCs were chosen for experimental use on the basis of several standard factors indicative of overall health. Selected cells had a uniform cylindrical shape with a basally located nucleus, no intracellular Brownian motion or other detectable cytosolic movement, and were used within 4 to $6 \mathrm{~h}$ of excision. In addition to these standard criteria, the use of di-8-ANEPPS allowed for an additional mechanism to select cells for FRAP experimentation. When externally applied, di-8-ANEPPS preferentially resides in the outer leaflet of cellular plasma membranes (Bedlack et al. 1992, 1994) and shows little internalization in healthy OHCs. Selected cells satisfied both brightfield and fluorescence criteria.

\section{Cholesterol manipulations in $\mathrm{OHCs}$}

Solutions to alter membrane cholesterol were made by diluting either methyl- $\beta$-cyclodextrin $(\mathrm{m} \beta \mathrm{CD})$ or water-soluble cholesterol $(\mathrm{m} \beta \mathrm{CD}+$ cholesterol; Sigma, St. Louis, MO, USA) in ECS. The osmolarity of these solutions was verified to be within 290-305 mOsm. OHCs were depleted using $0.05,0.1$, and $0.5 \mathrm{mM} \mathrm{m \beta CD}$ and loaded using $0.05,0.1,0.5$, and $1 \mathrm{mM} \mathrm{m} \beta \mathrm{CD}+$ 
cholesterol. All treatment concentrations are indicative of the final $\mathrm{m} \beta \mathrm{CD}$ concentration. In addition to various concentrations, two techniques were used to expose OHCs to cholesterol solutions, whole cochlea and isolated OHCs. For whole cochlea treatments, after di8-ANEPPS incubation, the bulla was transferred to the desired cholesterol solution for $10 \mathrm{~min}$ at room temperature. Then, the bulla was placed in fresh ECS and OHCs were dissociated and plated into MatTek dishes. For isolated OHC treatments, post-incubation in di-8-ANEPPS, OHCs were dissociated and plated in eight-well Lab-Tek chambered cover glass (Nalgene Nunc International, Naperville, IL, USA). Each well was then treated with the appropriate cholesterol solution for $10 \mathrm{~min}$. This method allowed direct access of cholesterol solutions to OHCs. Since there was no wash step after cholesterol manipulations in isolated OHCs, experiments were only performed on each chamber for $\leq 60 \mathrm{~min}$ after initial exposure to treatment solutions.

It should be noted that cholesterol manipulations in OHCs are challenging experiments. Treatment of OHCs can lead to a rapid lengthening and shortening of the cell before rupture of the plasma membrane and cell death (Rajagopalan et al. 2007). Extreme care was taken to observe cell health during the time course of FRAP experimentation. Here, the use of di8-ANEPPS as a probe was highly beneficial, as the appearance of any internal fluorescence indicated breakdown of the plasma membrane and the internalization of di-8-ANEPPS from the outer leaflet of the bilayer. For OHCs included in FRAP analysis, we measured the cell length and the mid-width during experimentation to confirm that cholesterol treatments did not affect morphology.

\section{Human embryonic kidney cell culture} and fluorescent labeling

HEK cells were cultured in Dulbecco's modified Eagle's medium (DMEM) with HEPES, $\mathrm{NaHCO}_{3}, 10 \%$ bovine calf serum, and 1\% Pen-Strep (cell media) under standard conditions of $37^{\circ} \mathrm{C}, 95 \%$ humidity, and $5 \%$ $\mathrm{CO}_{2}$. Cells were fed on alternate days and passaged at confluency, usually 5 to 7 days. Cells were plated $24-48 \mathrm{~h}$ prior to experimentation at a density of 500,000 $1,000,000$ cells/dish in MatTeks that were also coated with an additional layer of poly-D-lysine as described previously (Organ and Raphael 2007). HEKs were labeled with $40 \mu \mathrm{M}$ di-8-ANEPPS in cell media for $20 \mathrm{~min}$ at $37^{\circ} \mathrm{C}$.

\section{Cholesterol manipulations in HEKs}

Membrane cholesterol levels in HEKS were altered using 5 and $10 \mathrm{mM} \mathrm{m} \beta \mathrm{CD}$ or water-soluble cholesterol $(\mathrm{m} \beta \mathrm{CD}+$ cholesterol $)$ in DMEM. After
di-8-ANEPPS loading, cells were treated with cholesterol solutions for $10 \mathrm{~min}$ at $37^{\circ} \mathrm{C}$. After incubation, cells were rinsed once in phosphatebuffered saline (PBS) and placed in fresh PBS for FRAP experimentation.

\section{FRAP acquisition and analysis}

FRAP experiments were conducted on a Zeiss LSM 510 confocal microscope at room temperature as described previously (Organ and Raphael 2007). In brief, di-8-ANEPPS was excited with 488-nm light from a $30 \mathrm{~mW}$ argon $/ 2$ laser set at $50 \%$ output, corresponding to a tube current of 5.5-5.7 $\mathrm{A}$ and $1 \%$ transmission. Twelve-bit $512 \times 512$ images were obtained at a digital zoom of 2 and with a pixel dwell time of $1.60 \mu \mathrm{s}$. Images were formed confocally with a pinhole of 1 Airy unit, corresponding to an image depth of $<0.8 \mu \mathrm{m}$.

Each experiment consists of a time series of 410 scans of a $14.3-\mu \mathrm{m}^{2}$ region with a $200-\mathrm{ms}$ delay between scans. Within this area, we monitor five regions of interest (ROIs), each $2.9 \mu \mathrm{m}$ in diameter, representing a bleach ROI, two control ROIs, and a background ROI (Fig. 1B). A double bleaching protocol (Lippincott-Schwartz et al. 2003; Stavreva and McNally 2004; Organ and Raphael 2007) is used consisting of two successive bleaches, one after scan 10 and one after scan 210, each lasting less than $1 \mathrm{~s}$. A population of truly immobile molecules will be permanently photobleached during the first bleach so they will not add to the fluorescent signal monitored during the second bleach. Theoretically then, for untethered molecules, the mobile fraction from the second bleach should approach $100 \%$.

For FRAP analysis, the Zeiss AIM physiology software was used to quantify intensities, and the raw data were background-subtracted before each bleach and then individually normalized to the average of the first ten scans (scans 200-210 for the second bleach) to produce recovery curves. The normalized data were used to calculate the mobile fraction, $M_{\mathrm{f}}$, using Eq. 1, where $I_{\mathrm{i}}$ is the intensity of the first ten scans, equal to one after normalization; $I_{\mathrm{O}}$ is the intensity immediately post-bleach; and $I_{\infty}$ is the recovery plateau intensity, the average value of the last ten scans (Fig. 1C). To calculate effective diffusion coefficients, $D$, recovery curves were individually fit to the solution to the diffusion equation in one dimension based on the work of Axelrod et al. (1976) and Soumpasis (1983) and detailed in the Appendix.

$$
M_{f}=\left(\frac{I_{\infty}-I_{o}}{I_{i}-I_{o}}\right) \times 100 \%
$$




\section{Data set development}

Since few qualitative, and no quantitative, data were available in real time during FRAP experimentation, we developed a set of criteria which we implemented in the analysis phase to determine whether a particular recovery curve was included in the final data set. The FRAP parameters of bleaching efficiency or depth of bleaching, $B$, as well as the photobleaching due to repeated sample scanning, $\mathrm{PB}$, calculated from control regions, were used to select data for the final data pools (Lippincott-Schwartz et al. 1999; Meyvis et al. 1999; Snapp et al. 2003; Organ and Raphael 2007). Individual cells were included if $45 \leq B \leq 95 \%$, and the average values of $B$ for various cell types and treatments were between $64 \%$ and $92 \%$. Cells were eliminated if $\mathrm{PB}$ was above 25\%, and the average values for the two cell types and various treatments ranged between $7 \%$ and $15 \%$. The parameters $\mathrm{PB}, B$, and $M_{\mathrm{f}}$ were evaluated from normalized recovery curves, and experiments with $M_{\mathrm{f}}$ values greater than $100 \%$ were also discarded.

Recovery curves were then individually curve fit in Matlab (The MathWorks, Inc., Natick, MA, USA) with two free parameters: the effective diffusion coefficient, $D$, and a scaling coefficient to account for immobile fractions. The numerical fitting routine utilized 500 terms to represent the infinite sum (see Appendix), and no variation in $D$ values was seen when including between 50 and 1,000 terms. Occasionally, raw recovery curves had gross abnormalities and/or poor fits. To address these anomalous data, we applied Chauvenet's criterion, a statistical method of outlier detection, to all calculated $D$ values (Taylor 1997). From an initial pool of 158 cells, Chauvenet's criterion identified 12 cells as outliers, which were then removed from the final data set. Excluded experiments were evenly distributed across $\mathrm{OHC}$ regions and treatment groups and had an average $D$ value of $5.93 \mu \mathrm{m}^{2} / \mathrm{s}$, much larger than the typical diffusion coefficients measured. The average $R^{2}$ value of excluded cells was 0.56 , indicative of poor fits due to anomalies in the recovery curve shape. On average, excluded points were 2.5 standard deviations from the mean of their respective data set.

The final calculated $D$ values were not significantly different between bleaches based on two-factor analysis of variance (ANOVA). Bleach was a significant factor in two-factor ANOVA of $M_{\mathrm{f}}$ values, with the first bleach significantly lower than the second, indicating the elimination of an immobile pool of molecules during the first bleach. The values for $M_{\mathrm{f}}$ and $D$ reported are from the second bleach, and statistical significance was determined by ANOVA with Tukey honestly significance post hoc testing unless otherwise indicated. Values, in both text and tables, are reported as mean \pm $\mathrm{SD}$, and figures show data as mean \pm SEM.

\section{RESULTS}

\section{Regional differences in lateral mobility}

To examine potential variations in lateral mobility between $\mathrm{OHC}$ plasma membrane regions, we compared FRAP results of di-8-ANEPPS diffusion in each area (Fig. 2). $M_{\mathrm{f}}$ values were highest in the lateral region and were statistically different between all regions, measuring $94 \pm 5 \%, 85 \pm 9 \%$, and $76 \pm 11 \%$ for lateral, basal, and apical regions, respectively (Table 1 ). $D$ was significantly higher in the apical region than in the lateral and basal regions and was calculated as $5.26 \pm 3.95,1.67 \pm 0.89$, and $1.29 \pm 0.47 \mu \mathrm{m}^{2} / \mathrm{s}$, respectively (Fig. 3 and Table 1 ).

\section{Effect of cholesterol manipulations on regional lateral mobility in $\mathrm{OHCs}$}

Within each OHC region, membrane cholesterol levels were altered by either $0.5 \mathrm{mM}$ depletion or loading via the whole cochlea method. Manipulations of membrane cholesterol levels did not affect the overall morphology of OHCs. Cell length was measured after treatment, just prior to commencing a FRAP experiment, and OHC width was measured at the midpoint of the FRAP experiment. There were no changes in these parameters with treatments $(p>0.1)$, and the cumulative average values $(n=134)$ were $58 \pm 10$ and $10 \pm 1.1 \mu \mathrm{m}$ and $5.9 \pm 1.5$ for OHC length, mid-experiment width, and aspect ratio, respectively (data not shown).

While mobility parameters in the apical region were primarily unaffected by cholesterol manipulations, both the lateral and basal regions showed sensitivity to membrane cholesterol levels (Fig. 4 and Table 2). In the lateral region, $M_{\mathrm{f}}$ values were significantly decreased from control after cholesterol loading, dropping from $94 \pm 5 \%$ to $88 \pm 5 \%$. The effective diffusion coefficient of the lateral wall was also significantly decreased more than twofold after loading from $1.67 \pm 0.89$ to $0.81 \pm$ $0.42 \mu \mathrm{m}^{2} / \mathrm{s}$. Thus, cholesterol loading decreased mobility in the lateral region of OHCs.

In the basal region, while $M_{\mathrm{f}}$ values were unchanged upon cholesterol manipulations, cholesterol loading increased $D \sim 30 \%$ from a control value of $1.29 \pm 0.47$ to $1.64 \pm 0.54 \mu \mathrm{m}^{2} / \mathrm{s}$. Cholesterol depletion had the opposite effect in the basal region, reducing $D \sim 25 \%$ to $0.97 \pm 0.36 \mu \mathrm{m}^{2} / \mathrm{s}$, and the two treatments were statistically significant from one another. Thus, in the basal region, cholesterol depletion reduced mobility while loading increased it.

\section{Cholesterol-induced mobility changes} in the $\mathrm{OHC}$ lateral plasma membrane

Initial results in the lateral region demonstrated a significant decrease in mobility from control upon cholesterol loading (Fig. 4 and Table 2). This is 


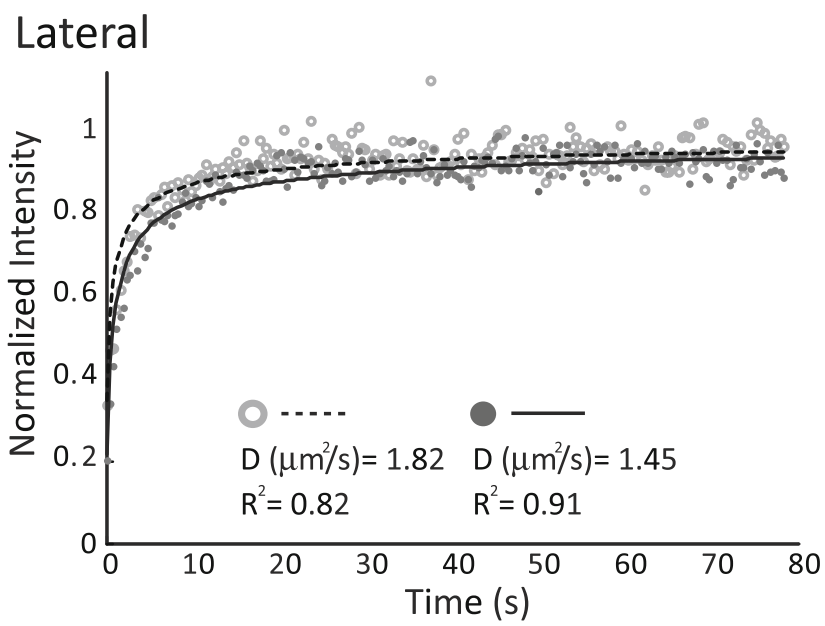

Basal

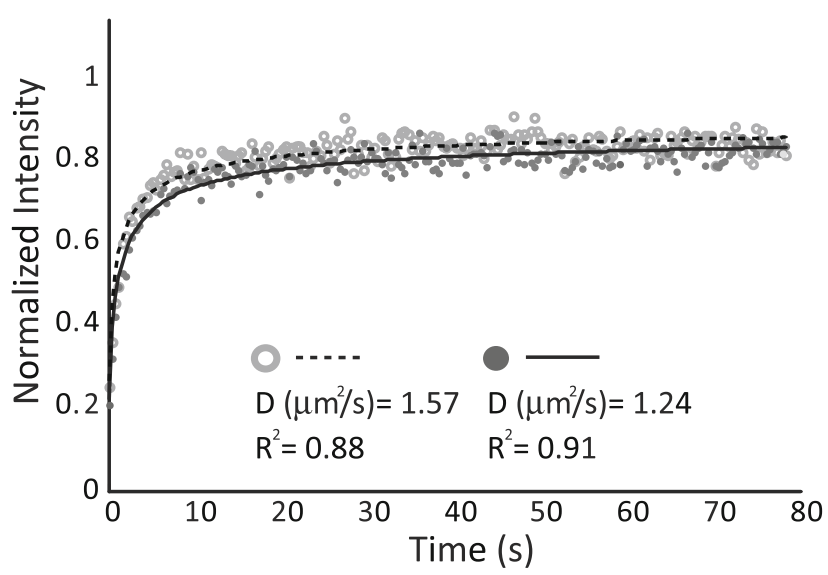

\section{Apical}

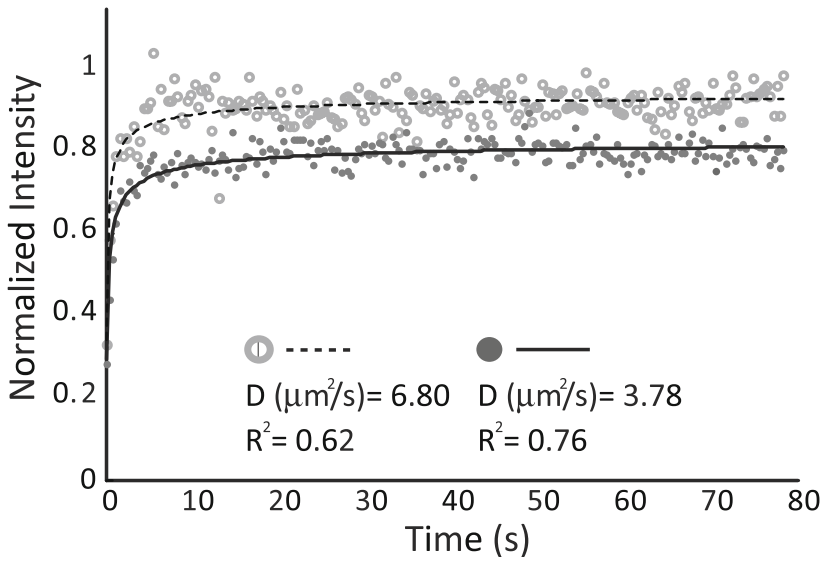

FIG. 2. Recovery data points and curve fits for six representative cells, two from each $\mathrm{OHC}$ plasma membrane region (lateral, basal, and apical). Data points have been background subtracted and normalized. Calculated effective diffusion coefficients, $D$, as well as goodness of fit parameters, $R^{2}$, are provided from individual curve fits of each cell. Data from the apical region show more scatter within individual experiments, a higher variability between experiments, and lower $R^{2}$ values than the lateral and basal membrane curves.

\begin{tabular}{lccc}
\hline \multicolumn{4}{c}{ TABLE 1 } \\
\hline \multicolumn{4}{c}{ Regional differences in lateral mobility in OHCs } \\
\hline & Lateral $(n=17)$ & Basal $(n=13)$ & Apical $(n=15)$ \\
\hline$M_{\mathrm{f}}(\%)$ & $94 \pm 5^{\mathrm{a}}$ & $85 \pm 9^{\mathrm{b}}$ & $76 \pm 11^{\mathrm{b}, \mathrm{a}}$ \\
$D\left(\mu \mathrm{m}^{2} / \mathrm{s}\right)$ & $1.67 \pm 0.89$ & $1.29 \pm 0.47$ & $5.26 \pm 3.95^{\mathrm{b}, \mathrm{a}}$ \\
$R^{2}$ & $0.83 \pm 0.09$ & $0.81 \pm 0.11$ & $0.56 \pm 0.17$ \\
\hline
\end{tabular}

Average values $\pm \mathrm{SD}$ for $M_{\mathrm{f}}$ and $D . R^{2}$ values for fits used to determine $D$ are provided. $D$ was similar between the lateral and basal regions but was statistically significantly higher in the apical region $(p<0.0005)$.

${ }^{\mathrm{a}} M_{\mathrm{f}}$ values were statistically different between all regions $(p<0.05)$ for comparison to basal

${ }^{b} M_{f}$ values were statistically different between all regions $(p<0.05)$ for comparison to lateral

particularly interesting as the $\mathrm{OHC}$ lateral wall is the predominant location of prestin and since alterations in membrane cholesterol have been shown to affect prestin function and electromotility (Rajagopalan et al. 2007). We further examined the effects of cholesterol manipulations on the lateral membrane by exploring additional treatment concentrations, and Figure 5A recasts the lateral region results of Figure 4 with the addition of a group of cells loaded at $0.1 \mathrm{mM}$. We also considered whether our method of bathing whole exposed cochlea in cholesterol solutions was limiting the exposure of individual OHCs, masking or hindering shifts in mobility parameters. Hence, we introduced an isolated $\mathrm{OHC}$ treatment protocol, where cholesterol solutions were directly applied to small numbers of individual cells, and observed significant decreases in mobility in the lateral membrane following both depletion and loading (Fig. 5B and Table 3). We also noted that treating isolated OHCs has a more significant effect than treating whole cochleae.

In isolated OHCs, only the strongest depletion concentration, $0.1 \mathrm{mM}$, significantly affected the $M_{\mathrm{f}}$ (Table 3). This depletion concentration also significantly reduced $D$ from $1.67 \pm 0.89$ to $0.54 \pm 0.18 \mu \mathrm{m}^{2} / \mathrm{s}$, a

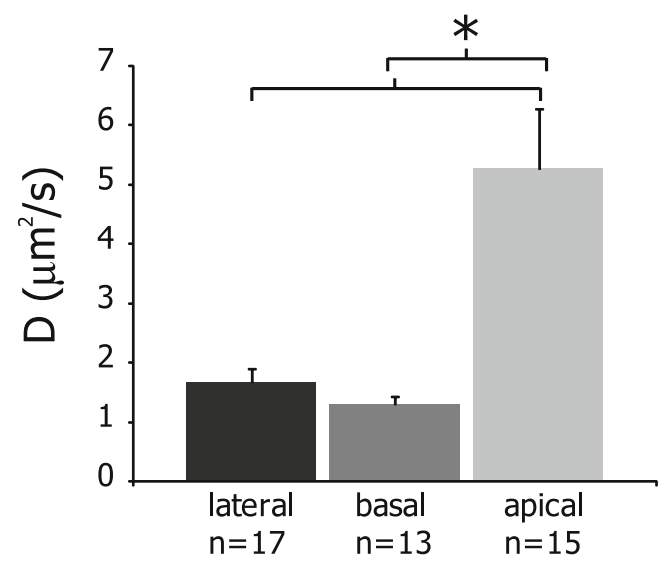

FIG. 3. Effective diffusion coefficients, $D$, across the three $\mathrm{OHC}$ regions. $D$ values in the apical region were statistically significantly larger than in the lateral or basal regions, as indicated by an asterisk $(p<0.0005)$. Values are plotted as mean \pm SEM. 

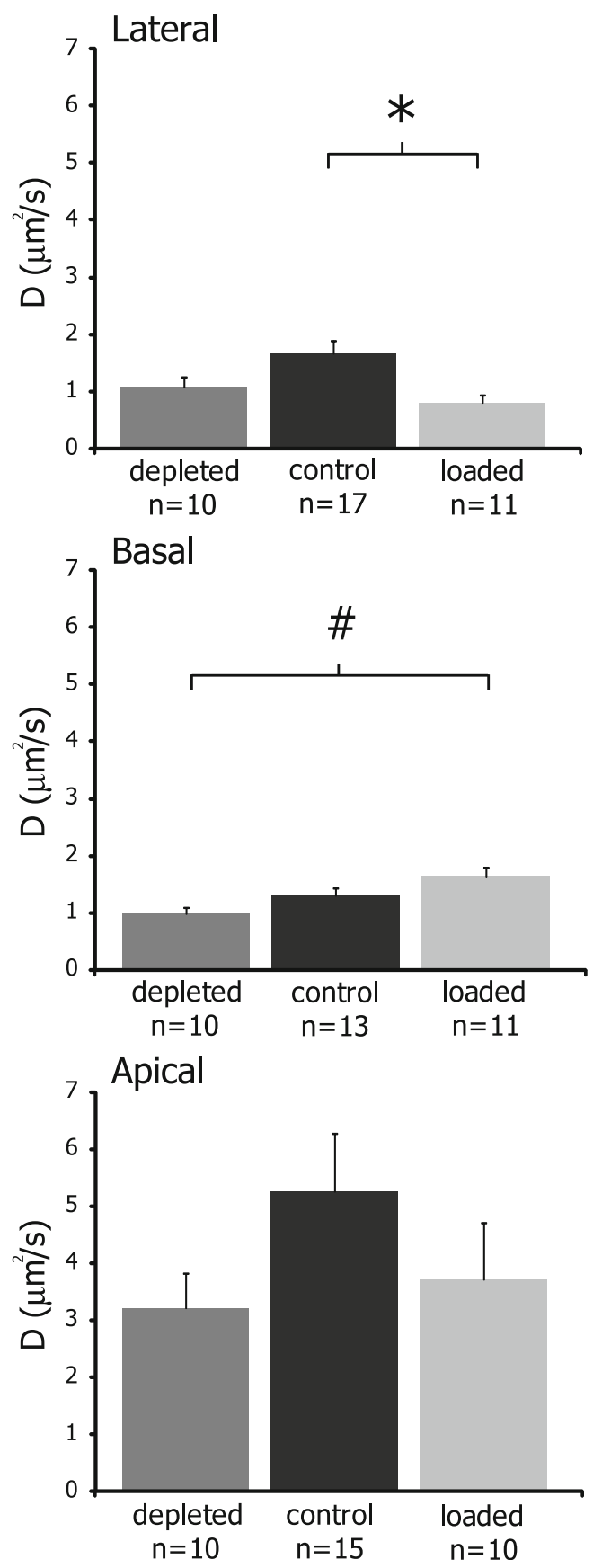

FIG. 4. Effects of cholesterol manipulations on the effective diffusion coefficients in the $\mathrm{OHC}$ lateral, basal, and apical regions. $D$ values were significantly altered from the control value following cholesterol loading in the lateral region $(p<0.01)$. In the basal region, depletion and loading had significantly different $D$ values $(p<0.01)$. No statistical differences were detected in the apical region $(p>0.3)$. Values are plotted as mean \pm SEM. Statistical significance from control values is indicated by an asterisk, while significance between loading and depletion is indicated by a number symbol.

more than threefold decrease. Cholesterol loading of isolated OHCs at $0.05,0.1$, and $1 \mathrm{mM}$ also significantly reduced $D$ values in the lateral membrane to $0.70 \pm 0.35$, $0.72 \pm 0.32$, and $0.29 \pm 0.08 \mu \mathrm{m}^{2} / \mathrm{s}$, respectively, representing a two to fivefold decrease.
Effect of cholesterol manipulations on lateral mobility in HEK cells

To examine whether the cholesterol-sensitive changes in lateral diffusion were specific to OHCs, we performed additional FRAP experiments on di-8ANEPPS labeled HEK cells treated with cholesterol altering solutions (Fig. 6 and Table 4). No statistically significant differences were seen in either $M_{\mathrm{f}}$ or $D$ upon cholesterol manipulations, although $10 \mathrm{mM}$ cholesterol loading did result in $\sim 25 \%$ increase in $D$ from $0.81 \pm 0.30$ to $1.01 \pm 0.42 \mu \mathrm{m}^{2} / \mathrm{s}$.

\section{DISCUSSION}

The material properties of plasma membranes play a crucial role in both normal and abnormal cellular physiology. Cholesterol is an essential component of the plasma membrane, and recent work highlights the importance of examining how alterations in cholesterol affect bilayer properties and the function and activity of membrane proteins (Yeagle 1991; Andersen and Koeppe 2007). In the auditory field, relatively few studies have addressed the role of membrane cholesterol in hair cell physiology, although recent studies have called attention to the importance of the membrane environment that surrounds and supports prestin. Like many membrane proteins, the mechanisms of prestin

TABLE 2

Mobility parameters in the three $\mathrm{OHC}$ regions after cholesterol manipulations

\begin{tabular}{lccc}
\hline & Depleted & Control & Loaded \\
\hline$M_{\mathrm{f}}(\%)$ & & & \\
Lateral & $90 \pm 6$ & $94 \pm 5^{\mathrm{a}}$ & $88 \pm 5^{\mathrm{a}}$ \\
Basal & $80 \pm 11$ & $85 \pm 9$ & $84 \pm 7$ \\
Apical & $72 \pm 12$ & $76 \pm 11$ & $79 \pm 4$ \\
$D\left(\mu \mathrm{m}^{2} / \mathrm{s}\right)$ & & & \\
Lateral & $1.08 \pm 0.60$ & $1.67 \pm 0.89^{\mathrm{a}}$ & $0.81 \pm 0.42^{\mathrm{a}}$ \\
$R^{2}$ & $0.84 \pm 0.10$ & $0.83 \pm 0.09$ & $0.87 \pm 0.08$ \\
Basal & $0.97 \pm 0.36^{\mathrm{b}}$ & $1.29 \pm 0.47$ & $1.64 \pm 0.54^{\mathrm{b}}$ \\
$R^{2}$ & $0.84 \pm 0.08$ & $0.81 \pm 0.11$ & $0.75 \pm 0.08$ \\
Apical & $3.22 \pm 1.93$ & $5.26 \pm 3.95$ & $3.71 \pm 3.16$ \\
$R^{2}$ & $0.60 \pm 0.14$ & $0.56 \pm 0.17$ & $0.58 \pm 0.21$ \\
\hline
\end{tabular}

Only the lateral region showed significant changes in $M_{\mathrm{f}}$ after cholesterol treatments with loading decreased from control $(p<0.05)$. The effective diffusion coefficient, $D$, of the lateral region significantly decreased after cholesterol loading $(p<0.01)$. In the basal region, cholesterol loading and depletion resulted in $D$ values that were statistically significant from one another $(p<0.01)$. No change was seen in the apical region $D$ values following cholesterol manipulations $(p>0.3)$. Values shown are mean $\pm S D$. In the lateral region, $n$ was 10,17 , and 11 for depleted, control and loaded, respectively. In the basal region, $n$ was 10,13 , and 11 , and in the apical region, $n$ was 10,15 , and $10 . R^{2}$ values demonstrating goodness of fit for calculations of $D$ are also provided

${ }^{\text {a }}$ Significant differences from control

${ }^{\mathrm{b}}$ Significant difference between loading and depletion 


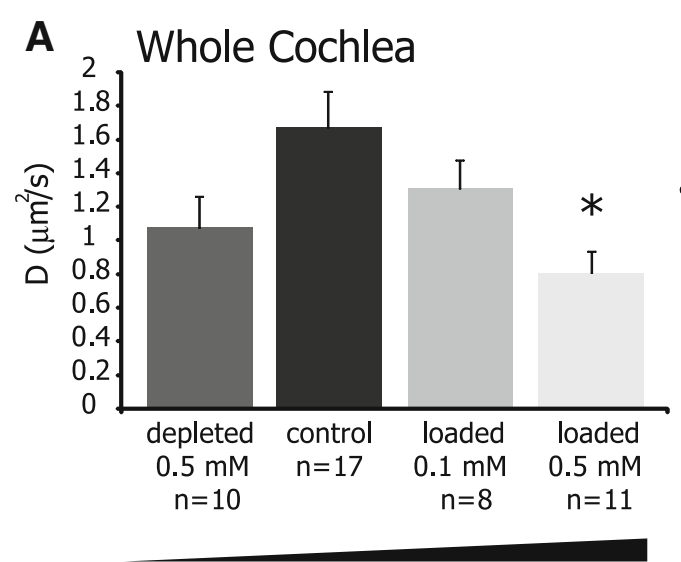

Increasing Cholesterol Concentration

\section{B Isolated $\mathrm{OHCs}$}

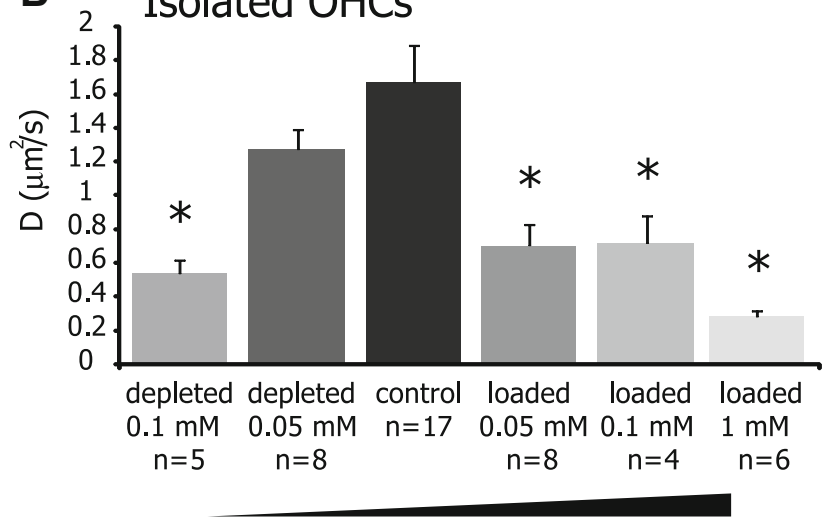

Increasing Cholesterol Concentration

FIG. 5. Effective diffusion coefficient measurements from the $\mathrm{OHC}$ lateral wall after various cholesterol manipulations. In $\mathbf{A}$, cholesterol treatments were applied to the whole cochlea, while in $\mathbf{B}$, only isolated $\mathrm{OHCs}$ were exposed. For both treatment techniques, cholesterol

function will not be fully understood in isolation from its interactions with the $\mathrm{OHC}$ plasma membrane.

To probe potential membrane-protein interactions in OHCs, we conducted FRAP experiments to determine whether lipid lateral mobility varies with OHC region and to investigate whether membrane fluidity is sensitive to manipulations in membrane cholesterol. We anticipated that plasma membrane material properties differ between the three OHC regions and that these properties affect lateral diffusion as experimentally measured with FRAP. After characterizing the regional lateral mobility of lipids in OHCs, we addressed whether altering the membrane cholesterol content modulates lipid lateral diffusion and concluded that the OHC lateral wall plasma membrane environment is dynamically tuned by cholesterol in a manner which may be functionally relevant for effective electromotility.

loading and depletion showed significant decreases in $D$ values as compared to control $(p<0.05)$. Values are plotted as mean \pm SEM, and statistically significant differences from control (indicated by an asterisk) are based on one-factor ANOVA with Dunnett's post hoc testing.

Our FRAP protocol for measuring di-8-ANEPPS lateral mobility in the $\mathrm{OHC}$ produced $D$ values that were comparable to those reported in other studies of di-8-ANEPPS diffusion in OHCs (Oghalai et al. 1999, 2000; de Monvel et al. 2006; Chen and Zhao 2007). Although our effective diffusion coefficients were somewhat larger, we attribute this to differences in experimental setup, diffusion models, and fitting routines, since our reported $D$ values for di-8-ANEPPS diffusion in HEKs and OHCs were similar. All values are within the range typically reported for macroscopic effective diffusion coefficients in cell membranes, on the order of $10^{-8}$ to $10^{-9} \mathrm{~cm}^{2} / \mathrm{s}$ (Edidin 1987; Fujiwara et al. 2002). However, comparisons of quantitative mobility parameters showed distinct differences in lateral mobility between OHC regions. Specifically, the mobile fraction $\left(M_{\mathrm{f}}\right)$ of di-8-ANEPPS in the lateral region was significantly higher than in the basal or

\section{TABLE 3}

\begin{tabular}{|c|c|c|c|c|c|c|}
\hline \multicolumn{7}{|c|}{ Mobility parameters in the $\mathrm{OHC}$ lateral plasma membrane after cholesterol manipulations } \\
\hline & Depleted & & Control & Loaded & & \\
\hline \multicolumn{7}{|l|}{ Whole cochlea } \\
\hline & $0.5 \mathrm{mM}(n=10)$ & & $(n=17)$ & $0.1 \mathrm{mM}(n=8)$ & & $0.5 \mathrm{mM}(n=11)$ \\
\hline$M_{\mathrm{f}}(\%)$ & $90 \pm 6$ & & $94 \pm 5$ & $93 \pm 7$ & & $88 \pm 5^{\mathrm{a}}$ \\
\hline$D\left(\mu \mathrm{m}^{2} / \mathrm{s}\right)$ & $1.08 \pm 0.60$ & & $1.67 \pm 0.89$ & $1.31 \pm 0.47$ & & $0.81 \pm 0.42^{\mathrm{a}}$ \\
\hline$R^{2}$ & $0.84 \pm 0.10$ & & $0.83 \pm 0.09$ & $0.87 \pm 0.10$ & & $0.87 \pm 0.08$ \\
\hline Isolated OHCs & $0.1 \mathrm{mM}(n=5)$ & $0.05 \mathrm{mM}(n=8)$ & & $0.05 \mathrm{mM}(n=8)$ & $0.1 \mathrm{mM}(n=4)$ & $1 \mathrm{mM}(n=6)$ \\
\hline$M_{\mathrm{f}}(\%)$ & $86 \pm 7^{a}$ & $94 \pm 3$ & $94 \pm 5$ & $91 \pm 6$ & $92 \pm 5$ & $87 \pm 6$ \\
\hline$D\left(\mu \mathrm{m}^{2} / \mathrm{s}\right)$ & $0.54 \pm 0.18^{\mathrm{a}}$ & $1.27 \pm 0.32$ & $1.67 \pm 0.89$ & $0.70 \pm 0.35^{\mathrm{a}}$ & $0.72 \pm 0.32^{\mathrm{a}}$ & $0.29 \pm 0.08^{a}$ \\
\hline$R^{2}$ & $0.95 \pm 0.02$ & $0.83 \pm 0.07$ & $0.83 \pm 0.07$ & $0.91 \pm 0.04$ & $0.94 \pm 0.02$ & $0.97 \pm 0.02$ \\
\hline
\end{tabular}

When examining the mobile fraction, only $0.5-\mathrm{mM}$ loading of whole cochlea and $0.1-\mathrm{mM}$ depletion of isolated $\mathrm{OHCs}$ showed reduction in $M_{\mathrm{f}}$ values that were different from control $(p<0.05)$. However, various loading and depletion treatments showed significant decreases in $D$ values compared to control ( $p<0.05)$. Values are shown as mean $\pm \mathrm{SD} . R^{2}$ values demonstrating goodness of fit for calculations of $D$ are also provided

${ }^{\text {a }}$ Statistically significant differences from control based on one-factor ANOVA with Dunnett's post hoc testing 


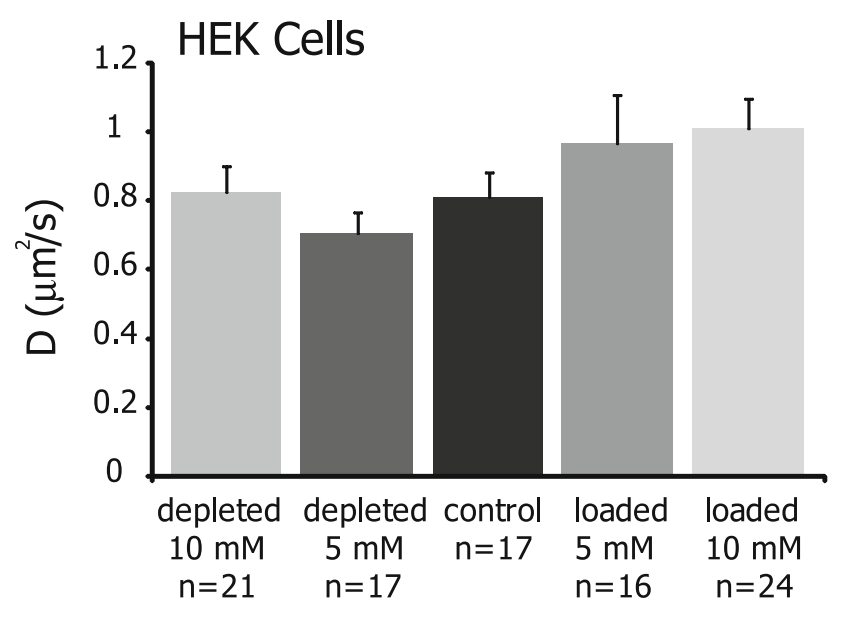

FIG. 6. Cholesterol manipulations do not significantly affect the lateral mobility of di-8-ANEPPS in HEK cells $(p>0.1)$, although cholesterol loading at 5 and $10 \mathrm{mM}$ results in a $\sim 20-25 \%$ increase in $D$. Values are shown as mean \pm SEM.

apical regions. Although calculated values of the effective diffusion coefficient were similar between the lateral and basal regions, $D$ was significantly larger, more than threefold, in the apical region. While all three OHC regions are highly specialized, in the apical and basal portions, there are physical connections to the surrounding cell types. As such, the isolation of OHCs necessary for FRAP analysis may induce changes in membrane properties in these two regions. While our methodology necessitates $\mathrm{OHC}$ isolation, lateral mobility has been preliminarily assessed in whole cochlear preparations (de Monvel et al. 2006), and future studies of OHCs would benefit from the development and expansion of techniques to examine the OHC plasma membrane in intact tissue.

FRAP experiments next examined the sensitivity of each region to membrane cholesterol depletion and loading. In the lateral region, cholesterol loading significantly lowered both the $M_{\mathrm{f}}$ and $D$, indicative of an overall decrease in lateral mobility. In the basal region, neither cholesterol treatment altered mobility parameters significantly from control values. However, depletion and loading caused significant shifts in $D$ values in opposite directions, making the two treatments statistically different from one another (Fig. 4 and Table 2). Thus, in the basal region, depletion led to a decrease in $D$, indicating a reduction in mobility, while loading raised $D$, indicating an increase in lateral mobility. These results are interesting given the lack of an obvious barrier separating the lateral and basal portions of the cell. Opposing effects of cholesterol treatments between the two regions suggest that the membrane properties of the OHC lateral wall and basal pole are significantly different, possibly as a result of dynamically maintained variations in lipid composition. Moreover, in di-8-ANEPPS-labeled HEK cells, cholesterol loading increased $D \sim 20-25 \%$, but the shift was not statistically significant. These results indicate that the fluidity of the OHC lateral membrane is highly sensitive to cholesterol manipulations.

The OHC apical region showed no significant changes upon cholesterol treatment, although both loading and depletion led to a reduction of $D$. However, measurements of FRAP parameters in the apical region had more variation than studies in either other OHC regions (Fig. 2) or HEKs. The standard deviations of $D$ values were large, and $R^{2}$ values for fits used to calculate $D$ were particularly low (Table 2). Additionally, the $M_{\mathrm{f}}$ values were consistently less than in the lateral or basal regions (Table 2). There are several possible sources of the large variations seen in FRAP measurements from the OHC apex. First, the $\mathrm{OHC}$ apical pole has been demonstrated to be the site of rapid membrane endocytosis possibly for both the recycling and sorting of membrane constituents (Meyer et al. 2001; Griesinger et al. 2004; Kaneko et al. 2006), and the presence and turnover of vesicles may contribute low levels of fluorescence that affect FRAP measurements. Second, the apical membrane geometry is more complex than either the lateral or basal regions. Although care was taken to place bleach ROIs so stereocilia were not included in the monitored region, di-8-ANEPPS molecules from the bundle will contribute to the recovery post-bleaching. Due to the unique cytoskeletal structure and regions of high curvature inherent to stereocilia, lipid diffusion in the apical region may be far more heterogeneous than in other areas of the OHC.

As a final study, we expanded our examination of the effects of cholesterol manipulations in the OHC lateral wall plasma membrane by performing experiments over a range of treatment concentrations. We were motivated by previous work demonstrating that cholesterol levels in the lateral plasma membrane

TABLE 4

\begin{tabular}{lccccc}
\hline \multicolumn{1}{c}{ TABLE 4 } \\
\hline \multicolumn{4}{c}{ Effect of cholesterol manipulations on lateral mobility parameters in HEK cells } \\
\hline & Depleted $10 \mathrm{mM}(n=21)$ & Depleted $5 \mathrm{mM}(n=17)$ & Control $(n=17)$ & Loaded $5 \mathrm{mM}(n=16)$ & Loaded 10 $\mathrm{mM}(n=24)$ \\
\hline$M_{\mathrm{f}}(\%)$ & $86 \pm 5$ & $86 \pm 6$ & $88 \pm 6$ & $87 \pm 9$ & $86 \pm 7$ \\
$D\left(\mu \mathrm{m}^{2} / \mathrm{s}\right)$ & $0.82 \pm 0.36$ & $0.70 \pm 0.26$ & $0.81 \pm 0.30$ & $0.96 \pm 0.57$ & $1.01 \pm 0.42$ \\
$R^{2}$ & $0.90 \pm 0.07$ & $0.94 \pm 0.04$ & $0.94 \pm 0.03$ & $0.91 \pm 0.09$ & $0.89 \pm 0.04$ \\
\hline
\end{tabular}

Membrane cholesterol manipulations do not produce significant changes to quantitative lateral mobility parameters in HEK cells $(p>0.1)$. Values shown are mean \pm SD. $R^{2}$ values demonstrating goodness of fit for calculations of $D$ are also provided 
alter prestin function and electromotility, as measured by NLC and DPOAEs (Rajagopalan et al. 2007). Cholesterol levels in the lateral wall also decrease during maturation from postnatal day 6 to adulthood, implying that membrane cholesterol is actively regulated by the OHC during development (Rajagopalan et al. 2007). Interestingly, while we did not see cholesterol sensitivity in our HEK lateral mobility measurements, Rajagopalan et al. did record directionally similar NLC shifts, a measure of prestin function, in both OHCs and prestin-expressing HEKs. However, the magnitude of cholesterol-induced effects was reduced in HEKs as compared to OHCs. Previous studies using fluorescent labels suggested that the cholesterol concentration of the OHC lateral plasma membrane is significantly less than in the apical or basal regions (Nguyen and Brownell 1998; Oghalai et al. 1998; Rajagopalan et al. 2007), so small changes in absolute cholesterol concentration in this region may have a significant effect on membrane material properties and membrane-protein interactions.

The results of further cholesterol manipulations in the OHC lateral plasma membrane showed insignificant changes in $M_{\mathrm{f}}$ values with treatment, but did demonstrate remarkable effects on $D$ values (Fig. 5 and Table 3 ). Both depletion and loading significantly reduced the effective diffusion coefficient in the OHC lateral region. Moreover, the effective diffusion coefficient is dependent on the lateral wall cholesterol concentration, both for treatments of whole cochleae and of isolated OHCs (Fig. 5 and Table 3). This indicates that cholesterol levels regulate the lipid lateral mobility and fluidity of the $\mathrm{OHC}$ lateral membrane; at the native cholesterol concentration, mobility is maximal, and perturbations in membrane cholesterol reduce lateral diffusion. Similar cholesterol manipulations have been shown to shift the voltage at which NLC is maximal, with depletion resulting in a depolarizing shift and loading leading to a hyperpolarizing shift (Rajagopalan et al. 2007). Although preliminary, this correlation between lipid lateral mobility, as an indicator of membrane fluidity, and shifts in the peak of nonlinear capacitance curves suggests that cholesterol-mediated tuning of prestin function and electromotility may result from membrane-protein interactions that are dynamically controlled by plasma membrane cholesterol levels.

Previous studies have shown that cholesterol regulates the function of other sensory and transport proteins, including rhodopsin (Boesze-Battaglia et al. 1989; Boesze-Battaglia and Albert 1990; Albert et al. 1996; Albert and Boesze-Battaglia 2005), the nicotinic acetylcholine receptor (Barrantes 2007), transient receptor proteins associated with cation channel complexes (Graziani et al. 2006), L-type calcium currents (Tsujikawa et al. 2008), as well as a variety of ion transporters and exchangers common to many cell types (Yeagle 1991; Bastiaanse et al. 1997; Andersen and Koeppe 2007). The suggested biophysical mechanism(s) responsible for these effects are diverse and include both specific lipid-protein interactions and more general membrane-protein interactions that either direct the lateral organization of proteins or modulate protein conformational changes (Yeagle 1991; Andersen and Koeppe 2007). Each specific protein transition has an associated energy cost which depends on the bilayer material properties, and changes in membrane fluidity may alter the kinetics of transitions between conformational states (Andersen and Koeppe 2007). Cholesterol-induced modulation of membrane properties has also been suggested to affect protein function through changes in the hydrophobic bilayer thickness, membrane curvature, lipid packing density, and the lateral pressure profile (Yeagle 1991; Cantor 1999; Lee 2003; Jensen and Mouritsen 2004; Mouritsen and Zuckermann 2004; McIntosh and Simon 2006; Andersen and Koeppe 2007; Niemela et al. 2007). Interestingly, material properties and cholesterol, in particular, can have either stimulatory or inhibitory effects on these proteins (Bastiaanse et al. 1997). Functional assays of several membrane transporters and exchangers show a biphasic activity profile as a function of membrane properties and cholesterol concentration (Yeagle 1991; Andersen and Koeppe 2007). Both lowering and raising the cholesterol levels from the native state result in alterations of membrane protein function, similar to what has been reported from NLC measurements in prestin-expressing cells and OHCs (Rajagopalan et al. 2007; Sfondouris et al. 2008).

However, it must be noted that the physiologic roles of cholesterol are numerous, complex, and difficult to analyze, as cholesterol simultaneously affects many interconnected membrane material properties on both a molecular and cellular scale (Yeagle 1991; Andersen and Koeppe 2007). It may be that cells maintain plasma membrane cholesterol levels in a constant balance between opposing effects on bilayer properties and/or lateral organization within the membrane plane (Yeagle 1991; Mouritsen and Zuckermann 2004). Thus, while translational diffusion in the lateral wall also shows a non-monotonic response to cholesterol levels, it is difficult to postulate a precise mechanism responsible for this effect. It is plausible that different mechanisms affect lipid mobility in a low cholesterol and high cholesterol environment (Yeagle 1991; Mouritsen and Zuckermann 2004) or that cholesterol loading has a different effect and mechanism than depletion, as has been recently suggested to explain the effects on prestin function (Sfondouris et al. 2008).

In summary, our data support the emerging idea that prestin function is intimately linked to membrane material properties. In general, our observations are consistent with a wealth of evidence demonstrating that 
a variety of manipulations which alter membrane material properties, including changes in cholesterol levels, also affect prestin function and/or electromotility (Kakehata and Santos-Sacchi 1995, 1996; Tunstall et al. 1995; Raphael et al. 2000; Lue et al. 2001; Santos-Sacchi and Wu 2004; Ermilov et al. 2005; Murdock et al. 2005; Spector et al. 2006; Fang and Iwasa 2007; Rajagopalan et al. 2007; Sturm et al. 2007; Ashmore 2008; Sfondouris et al. 2008). These studies have established that changes in membrane tension, stiffness, curvature, and lipid packing density all shift the voltage dependence of prestinassociated charge movement. Importantly, changes in membrane tension induced by osmotic challenge and amphipathic compounds, which also modify OHC membrane curvature and lipid packing density, significantly alter both NLC and lipid lateral diffusion in OHCs (Iwasa 1993; Kakehata and Santos-Sacchi 1995; Oghalai et al. 2000; Fang and Iwasa 2007; Greeson and Raphael 2009), providing precedent for a link between lipid mobility, changes in membrane properties, and prestin function. Future work is needed to further investigate the composition, organization, and dynamics of the OHC plasma membrane and to advance our understanding of the mechanisms by which the membrane environment is intricately involved in the regulation and modulation of prestin function and $\mathrm{OHC}$ electromotility.

\section{ACKNOWLEDGMENTS}

This work was supported by an NIH-NIDCD NRSA predoctoral fellowship F31 DC-08058-01 (L.E. Organ). Additional funding was provided by NIH-NIDCD (DC008134), NSF CAREER (BES no. 044379), and NSF MRI (BES no. 0321275) grants (R.M. Raphael). Special thanks to Dr. Imran Quraishi, Athanasios Dousis, and Dr. Eric Darling for assistance with data analysis, diffusion models, and Matlab programming, respectively. The authors would also like to thank Drs. Jennifer N. Greeson and Fred A. Pereira for their thoughtful commentary and expertise.

\section{APPENDIX}

To calculate effective diffusion coefficients, $D$, we fit normalized recovery curves to the $1 \mathrm{D}$ solution of the diffusion equation averaged over a line coincident with the membrane which encompasses the bleach ROI. Specifically, we model the diffusion of bleached molecules (Soumpasis 1983) on a line segment of length, $L$, the average perimeter of an particular cell type (Fig. 7A). This representative length encompassed the bleach segment, $a<x<b$, which is of length $\ell$, the diameter of the bleach ROI circle $(\sim 2.9 \mu \mathrm{m})$, and is centered within $L$. Using 26 HEKs, we calculated $L$ to be $112 \pm 32 \mu \mathrm{m}$ (mean \pm SD), and from 12 OHCs, we determined $L$ to be $130 \pm 21 \mu \mathrm{m}($ mean \pm SD) .
A

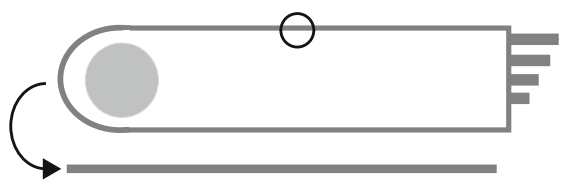

B

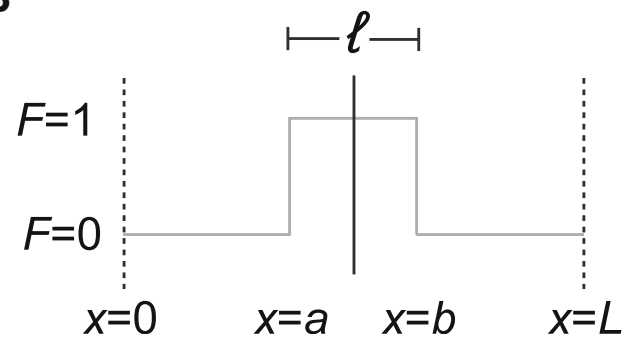

FIG. 7. The FRAP model implemented to calculate effective diffusion coefficients is based on 1D diffusion along a line with a set, characteristic length. This length, outlined in $\mathbf{A}$, is defined as the average perimeter of an outer hair cell, and is represented by $L$ in $\mathbf{B}$. The circle in $\mathbf{A}$ represents the bleach ROI, which has a diameter of $\sim 2.9 \mu \mathrm{m}$ and corresponds to $\ell$ in $\mathbf{B}$. The boundary conditions and variables used are shown in $\mathbf{B}$.

Beginning with a general form of the diffusion equation in $1 \mathrm{D}$ :

$$
\frac{\partial F(x, t)}{\partial t}=D \frac{\partial^{2} F(x, t)}{\partial x^{2}}
$$

Applying a conservation of mass:

$$
F_{\text {unbleached }}=1-F_{\text {bleached }} \text {. }
$$

Defining the boundary and initial conditions:

$F(0, t)=0$

$F(L, t)=0$

$F(x, 0)=1$ on $a<x<b$, where $a=\frac{L}{2}-\frac{\ell}{2}$, and $b=\frac{L}{2}+\frac{\ell}{2}$, as shown in Figure 7.

$F(x, 0)=0$ elsewhere outside this region, where $t(0)$ is immediately post-bleach.

Then, the general solution is of the form:

$$
F(x, t)=\sum_{n=1}^{\infty} c_{n} \sin \left(\frac{n \pi x}{L}\right) e^{-D \frac{n^{2} \pi^{2}}{L^{2}} t}
$$

Applying the initial condition:

$$
\begin{aligned}
c_{m} & =\frac{2}{L} \int_{0}^{L} \sin \left(\frac{m \pi x}{L}\right) F(x, 0) d x \\
c_{m} & =\frac{2}{L} \int_{a}^{b} \sin \left(\frac{m \pi x}{L}\right) d x \\
c_{m} & =\frac{-2}{m \pi}\left[\cos \left(\frac{m \pi b}{L}\right)-\cos \left(\frac{m \pi a}{L}\right)\right] \\
F(x, t) & =\sum_{n=1}^{\infty}\left(\frac{-2}{n \pi}\right)\left[\cos \left(\frac{n \pi b}{L}\right)-\cos \left(\frac{n \pi a}{L}\right) \sin \left(\frac{n \pi x}{L}\right) e^{-D \frac{n^{2} \pi^{2}}{L^{2}} t}\right]
\end{aligned}
$$

As the Zeiss physiology software quantifies the intensity at each time point as the average intensity in the bleach 
ROI, the solution was averaged over the bleach region $a<x<b$.

$$
F_{\text {avg }}(t)=\frac{1}{b-a} \int_{a}^{b} \sum_{n=1}^{\infty}\left(\frac{-2}{n \pi}\right)\left[\cos \left(\frac{n \pi b}{L}\right)-\cos \left(\frac{n \pi a}{L}\right)\right] \sin \left(\frac{n \pi x}{L}\right) e^{-D \frac{n^{2} \pi^{2}}{L^{2}}} d x
$$

Finally, the conservation of mass stated above is applied to develop an expression for the unbleached molecules.

$$
F_{\text {avg,unbleached }}(t)=1-\left(\frac{1}{b-a}\right) \sum_{n=1}^{\infty}\left(\frac{2 L}{n^{2} \pi^{2}}\right)\left[\cos \left(\frac{n \pi b}{L}\right)-\cos \left(\frac{n \pi a}{L}\right)\right]^{2} e^{-D^{n^{2} \pi^{2}} L^{2} t}
$$

\section{REFERENCES}

Albert AD, Boesze-Battaglia K. The role of cholesterol in rod outer segment membranes. Prog. Lipid Res. 44(2-3):99-124, 2005.

Albert AD, Boesze-Battaglia K, Paw Z, Watts A, Epand RM. Effect of cholesterol on rhodopsin stability in disk membranes. Biochim. Biophys. Acta 1297(1):77-82, 1996.

Andersen OS, Koepre RE, II. Bilayer thickness and membrane protein function: an energetic perspective. Annu. Rev. Biophys. Biomol. Struct. 36:107-130, 2007.

Ashmore J. Cochlear outer hair cell motility. Physiol. Rev. 88(1):173210, 2008.

Axelrod D, Koppel DE, Schlessinger J, Elson E, Webb WW. Mobility measurement by analysis of fluorescence photobleaching recovery kinetics. Biophys. J. 16(9):1055-1069, 1976.

BARRANTES FJ. Cholesterol effects on nicotinic acetylcholine receptor. J. Neurochem. 103(Suppl 1):72-80, 2007.

BastiaAnse EM, Hold KM, Van Der LaArse A. The effect of membrane cholesterol content on ion transport processes in plasma membranes. Cardiovasc. Res. 33(2):272-283, 1997.

BEDLACK RS, JR., WEI M, LOEW LM. Localized membrane depolarizations and localized calcium influx during electric field-guided neurite growth. Neuron. 9(3):393-403, 1992.

Bedlack RS, Jr., Wei MD, Fox SH, Gross E, Loew LM. Distinct electric potentials in soma and neurite membranes. Neuron. 13 (5):1187-1193, 1994.

Boesze-Battaglia K, Albert AD. Cholesterol modulation of photoreceptor function in bovine retinal rod outer segments. J. Biol. Chem. 265(34):20727-20730, 1990.

Boesze-Battaglia K, Hennessey T, Albert AD. Cholesterol heterogeneity in bovine rod outer segment disk membranes. J. Biol. Chem. 264 (14):8151-8155, 1989.

Brownell We, Spector AA, Raphael RM, Popel AS. Micro- and nanomechanics of the cochlear outer hair cell. Annu. Rev. Biomed. Eng. 3:169-194, 2001.

CANTOR RS. Lipid composition and the lateral pressure profile in bilayers. Biophys. J. 76(5):2625-2639, 1999.

Chen GD, Zhao HB. Effects of intense noise exposure on the outer hair cell plasma membrane fluidity. Hear. Res. 226(1-2):14-21, 2007.

Cullis PR, Fenske DB, Hope MJ. Physical properties and functional roles of lipids in membranes. In: Vance DE, Vance JE (eds) Biochemistry of Lipids, Lipoproteins, and Membranes. The Netherlands, Elsevier Science, 1996. de Monvel JB, Brownell WE, Ulfendahl M. Lateral diffusion anisotropy and membrane lipid/skeleton interaction in outer hair cells. Biophys. J. 91 (1):364-381, 2006.

EDIDIN M. Rotational and lateral diffusion of membrane proteins and lipids: phenomena and function. In: Bronner F, Kleinzeller A (eds) Current Topics in Membranes and Transport. New York, Academic, pp. 91-127, 1987.

EDIDIN M. Fluorescence photobleaching and recovery, FPR, in the analysis of membrane structure and dynamics. In: Damjanonich S, Edidin M, Szollosi J (eds) Mobility and Proximity in Biological Membranes. Boca Raton, FL, CRC, pp. 109-135, 1994.

Edidin M. Lipids on the frontier: a century of cell-membrane bilayers. Nat. Rev. Mol. Cell. Biol. 4(5):414-418, 2003.

Ermilov SA, Murdock DR, El-Daye D, Brownell WE, Anvari B. Effects of salicylate on plasma membrane mechanics. J. Neurophysiol. 94(3):2105-2110, 2005.

FANG J, IWASA KH. Effects of chlorpromazine and trinitrophenol on the membrane motor of outer hair cells. Biophys. J. 93(5):18091817, 2007.

Fujiwara T, Ritchie K, Murakoshi H, Jacobson K, Kusumi A. Phospholipids undergo hop diffusion in compartmentalized cell membrane. J. Cell Biol. 157(6):1071-1081, 2002.

Graziani A, Rosker C, Kohlwein SD, Zhu MX, Romanin C, Sattler W, Groschner K, Poteser M. Cellular cholesterol controls TRPC3 function: evidence from a novel dominant-negative knockdown strategy. Biochem. J. 396(1):147-155, 2006.

Greeson JN, Raphael RM. Application of fluorescence polarization microscopy to measure fluorophore orientation in the outer hair cell plasma membrane. J. Biomed. Opt. 12(2):021002, 2007.

Greeson JN, Raphael RM. Amphipath-induced nanoscale changes in outer hair cell plasma membrane curvature. Biophys. J. 96 (2):510-520, 2009.

Griesinger CB, Richards CD, Ashmore JF. Apical endocytosis in outer hair cells of the mammalian cochlea. Eur. J. Neurosci. 20(1):4150, 2004.

He DZ, Zheng J, Kalinec F, Kakehata S, Santos-Sacchi J. Tuning in to the amazing outer hair cell: membrane wizardry with a twist and shout. J. Membr. Biol. 209(2-3):119-134, 2006.

Heidemann SR, Wirtz D. Towards a regional approach to cell mechanics. Trends Cell Biol. 14(4):160-166, 2004.

Hilgemann DW. Getting ready for the decade of the lipids. Annu. Rev. Physiol. 65:697-700, 2003.

IwASA KH. Effect of stress on the membrane capacitance of the auditory outer hair cell. Biophys. J. 65(1):492-498, 1993. 
Janmey PA, Kinnunen PK. Biophysical properties of lipids and dynamic membranes. Trends Cell Biol. 16(10):538-546, 2006.

Jensen MO, Mouritsen OG. Lipids do influence protein function-the hydrophobic matching hypothesis revisited. Biochim. Biophys. Acta. 1666(1-2):205-226, 2004.

Kakehata S, Santos-Sacchi J. Membrane tension directly shifts voltage dependence of outer hair cell motility and associated gating charge. Biophys. J. 68(5):2190-2197, 1995.

Kakehata S, Santos-Sacchi J. Effects of salicylate and lanthanides on outer hair cell motility and associated gating charge. J. Neurosci. 16(16):4881-4889, 1996.

Kalinec F, Holley MC, Iwasa KH, Lim DJ, Kachar B. A membranebased force generation mechanism in auditory sensory cells. Proc. Natl. Acad. Sci. U. S. A 89(18):8671-8675, 1992.

Kaneko T, Harasztosi C, Mack AF, Gummer AW. Membrane traffic in outer hair cells of the adult mammalian cochlea. Eur. J. Neurosci. 23(10):2712-2722, 2006.

LEE AG. Lipid-protein interactions in biological membranes: a structural perspective. Biochim. Biophys. Acta 1612(1):1-40, 2003.

Lippincott-Schwartz J, Altan-Bonnet N, Patterson GH. Photobleaching and photoactivation: following protein dynamics in living cells. Nat. Cell Biol. Suppl:S7-14, 2003.

Lippincott-Schwartz J, Presley JF, Zaal KJ, Hirschberg K, Miller CD, ELLENBERG J. Monitoring the dynamics and mobility of membrane proteins tagged with green fluorescent protein. Methods Cell Biol. 58:261-281, 1999.

Lue AJ, Zhao HB, Brownell WE. Chlorpromazine alters outer hair cell electromotility. Otolaryngol. Head Neck Surg. 125(1):71-76, 2001.

Lundbaer Ja, Birn P, Hansen AJ, Sogaard R, Nielsen C, Girshman J, Bruno MJ, Tape Se, Egebjerg J, Greathouse DV, Mattice GL, Koeppe RE, 2ND, ANDERSEN OS. Regulation of sodium channel function by bilayer elasticity: the importance of hydrophobic coupling. Effects of Micelle-forming amphiphiles and cholesterol. J. Gen. Physiol. 123(5):599-621, 2004.

Marcucci R, Alessandrello liotta A, Cellai AP, Rogolino A, Berloco P, Leprini E, Pagnini P, Abbate R, Prisco D. Cardiovascular and thrombophilic risk factors for idiopathic sudden sensorineural hearing loss. J. Thromb. Haemost. 3(5):929-934, 2005.

McIntosh TJ, Simon SA. Roles of bilayer material properties in function and distribution of membrane proteins. Annu. Rev. Biophys. Biomol. Struct. 35:177-198, 2006.

Meyer J, Mack AF, Gummer AW. Pronounced infracuticular endocytosis in mammalian outer hair cells. Hear. Res. 161(1-2):10-22, 2001.

Meyvis TK, De Smedt SC, Van Oostveldt P, Demeester J. Fluorescence recovery after photobleaching: a versatile tool for mobility and interaction measurements in pharmaceutical research. Pharm. Res. 16(8):1153-1162, 1999.

Morizono T, Paparella MM. Hypercholesterolemia and auditory dysfunction. Experimental studies. Ann. Otol. Rhinol. Laryngol. 87(6 Pt 1):804-814, 1978.

Morizono T, Sikora MA, Ward WD, Paparella MM, Jorgensen J. Hyperlipidemia and noise in the chinchilla. Acta. Otolaryngol. 99(5-6):516-524, 1985.

Mouritsen OG, ZuCKermann MJ. What's so special about cholesterol? Lipids. 39(11):1101-1113, 2004.

Murdock DR, Ermilov SA, Spector AA, Popel AS, Brownell WE, ANVARI B. Effects of chlorpromazine on mechanical properties of the outer hair cell plasma membrane. Biophys. J. 89(6):40904095, 2005.

NeEdham D, Nunn RS. Elastic deformation and failure of lipid bilayer membranes containing cholesterol. Biophys. J. 58 (4):997-1009, 1990.
NGuYen TV, Brownell WE. Contribution of membrane cholesterol to outer hair cell lateral wall stiffness. Otolaryngol. Head Neck Surg. 119(1):14-20, 1998.

Niemela PS, Ollila S, Hyvonen MT, Karttunen M, Vattulainen I. Assessing the nature of lipid raft membranes. PLoS Comput. Biol. 3(2):e34, 2007.

Oghalai JS, Patel aA, Nakagawa T, Brownell WE. Fluorescenceimaged microdeformation of the outer hair cell lateral wall. J. Neurosci. 18(1):48-58, 1998.

Oghalai JS, Tran TD, Raphael RM, Nakagawa T, Brownell WE. Transverse and lateral mobility in outer hair cell lateral wall membranes. Hear. Res. 135(1-2):19-28, 1999.

Oghalai JS, Zhao HB, Kutz JW, Brownell WE. Voltage- and tensiondependent lipid mobility in the outer hair cell plasma membrane. Science. 287(5453):658-661, 2000.

Organ LE, Raphael RM. Application of fluorescence recovery after photobleaching to study prestin lateral mobility in the human embryonic kidney cell. J. Biomed. Opt. 12(2):021003, 2007.

Preyer S, Baisch A, Bless D, Gummer AW. Distortion product otoacoustic emissions in human hypercholesterolemia. Hear. Res. 152(1-2):139-151, 2001.

Rajagopalan L, Greeson JN, Xia A, Liu H, Sturm A, Raphael RM, Davidson AL, Oghalai JS, Pereira FA, Brownell WE. Tuning of the outer hair cell motor by membrane cholesterol. J. Biol. Chem. 282(50):36659-36670, 2007.

Raphael RM, Popel AS, Brownell WE. A membrane bending model of outer hair cell electromotility. Biophys. J. 78(6):2844-2862, 2000.

SaIto T, SATO K, Sarto H. An experimental study of auditory dysfunction associated with hyperlipoproteinemia. Arch. Otorhinolaryngol. 243 (4):242-245, 1986.

SANTOS-SACCHI J. New tunes from Corti's organ: the outer hair cell boogie rules. Curr. Opin. Neurobiol. 13(4):459-468, 2003.

SAntos-SAcchi J, WU M. Protein- and lipid-reactive agents alter outer hair cell lateral membrane motor charge movement. J. Membr. Biol. 200(2):83-92, 2004.

Schmidt D, Mackinnon R. Voltage-dependent $\mathrm{K}^{+}$channel gating and voltage sensor toxin sensitivity depend on the mechanical state of the lipid membrane. Proc. Natl. Acad. Sci. U. S. A. 105:1927619281, 2008.

Sfondouris J, Rajagopalan L, Pereira FA, Brownell We. Membrane composition modulates prestin-associated charge movement. J. Biol. Chem. 283(33):22473-22481, 2008.

Sikora MA, Morizono T, Ward WD, Paparella MM, Leslie K. Dietinduced hyperlipidemia and auditory dysfunction. Acta Otolaryngol. 102(5-6):372-381, 1986.

Snapp EL, Altan N, Lippincott-Schwartz J. Measuring protein mobility by photobleaching GFP chimeras in living cells. Curr Protoc Cell Biol. Chapter 21:Unit 21.1, 2003.

Soumpasis DM. Theoretical analysis of fluorescence photobleaching recovery experiments. Biophys J. 41(1):95-97, 1983.

Spector Aa, Deo N, Grosh K, Ratnanather JT, Raphael RM. Electromechanical models of the outer hair cell composite membrane. J. Membr. Biol. 209(2-3):135-152, 2006.

Stavreva DA, McNally JG. Fluorescence recovery after photobleaching (FRAP) methods for visualizing protein dynamics in living mammalian cell nuclei. Methods Enzymol. 375:443-455, 2004.

Sturm AK, Rajagopalan L, Yoo D, Brownell WE, Pereira FA. Functional expression and microdomain localization of prestin in cultured cells. Otolaryngol. Head Neck Surg. 136(3):434-439, 2007.

Takahashi S, Santos-Sacchi J. Non-uniform mapping of stressinduced, motility-related charge movement in the outer hair cell plasma membrane. Pflugers Arch. 441(4):506-513, 2001.

TAYLOR J. An Introduction to Error Analysis: The Study of Uncertainties in Physical Measurements. Sausalito, CA, University Science Books, 1997. 
Tsujikawa H, Song Y, Watanabe M, Masumira H, Gupte SA, Ochi R, OкADA T. Cholesterol depletion modulates basal L-type $\mathrm{Ca}^{2+}$ current and abolishes its -adrenergic enhancement in ventricular myocytes. Am. J. Physiol. Heart Circ. Physiol. 294(1):H285-H292, 2008.

Tunstall MJ, Gale JE, Ashmore JF. Action of salicylate on membrane capacitance of outer hair cells from the guinea-pig cochlea. J. Physiol. 485( Pt 3):739-752, 1995.
YeAgLe PL. Modulation of membrane function by cholesterol. Biochimie 73(10):1303-1310, 1991.

Zhang M, Kalinec F. Structural microdomains in the lateral plasma membrane of cochlear outer hair cells. J. Assoc. Res. Otolaryngol 3(3):289-301, 2002.

Zheng J, Shen W, He DZ, Long KB, Madison LD, Dallos P. Prestin is the motor protein of cochlear outer hair cells. Nature. 405 (6783):149-155, 2000. 